\title{
Tones and Numbers: Specificity of Interference In Immediate Memory
}

Abstract. Recognition of the pitch of a tone was severely disrupted by the incorporation of six other tones during a 5-second retention interval, even though the intervening tones could be ignored. However, the requirement to recall six numbers spoken at equal loudness during the identical retention interval produced only a minimum decrement in the same pitch-recognition task. Further, the requirement to remember the tone produced no decrement in recall of the numbers. It is concluded that immediate memory for pitch is subject to a large interference effect which is highly specific in nature and which is not due to some limitation in general short-term memory capacity or to a distraction of attention.

Interest has recently developed in the storage of unlabeled sensory information, and its interaction in memory with verbal materials. It has been proposed that sensory information is stored in a separate system, where it is subject to rapid decay but not to interference (1). Although a highly specific interference effect in visual memory has been reported (2), some have argued that this may be perceptual rather than mnemonic (3). It has also been speculated that the retention of sensory information in immediate memory requires the use of a limited central processing mechanism, and should therefore be disrupted by an intervening verbal memory task (4).

The experiment reported here demonstrates that memory for one kind of sensory information, in this case pitch, is subject to a large interference effect which is highly specific in nature. In all conditions, subjects listened to a 200-msec test tone, which was followed $5 \mathrm{sec}-$ onds later by another 200-msec test tone; and they judged whether the two were the same or different in pitch. In condition A, six extra tones of equal loudness were played during the interval between test tones. These were also $200 \mathrm{msec}$ in duration, and separated by intervals of $300 \mathrm{msec}$, leaving a 2-second pause before the second test tone. In conditions B, C, and D, six spoken numbers were incorporated during the interval between test tones. These were of equal loudness to the tones, and spaced identically. Subjects were instructed, in condition A, to ignore the intervening tones, and simply to indicate whether the test tones were the same or different in pitch by writing "S" or " $\mathrm{D}$." In condition B, they were similarly instructed to ignore the numbers and to compare the pitch of the test tones. In condition $\mathrm{C}$, subjects were required, in addition to comparing the tones, to recall the six numbers in their correct order. Having heard the entire sequence they wrote "S" or "D," followed by the numbers. In condition $\mathrm{D}$, the pitch of the test tones was always the same, and the subjects were informed of this. They were instructed to listen to the total sequence and then to write " $\mathrm{S}$ " followed by the numbers in their correct order.

There were 12 judgments to be made in each condition, separated by 20 -second pauses, which gave the sub- jects ample time to write their responses. Since there are 24 permutations of 4 conditions, 24 subjects were used, and each was given the conditions in a unique order. The subjects were selected on the basis of scoring 100 percent correct on a tape containing 12 pairs of test tones with 5-second intervals between pairs.

Tones were generated by a Wavetek oscillator controlled by a PDP 9 computer, and were recorded on tape; the numbers were then recorded on the same tape. Twelve tonal pitches were used. These were taken from an equal-tempered scale, ranging from the $\mathrm{C \#}$ a semitone above middle $\mathrm{C}$ to the $\mathrm{C}$ an octave above (5). The test tones differed in pitch in six examples in each condition, and always by a semitone (higher in half of the instances and lower in the other half). All pitches of test tones were equally represented in all conditions. The intervening tones were chosen randomly, except that the test tone pitches on any given trial were excluded. The intervening numbers, which ranged from 1 to 12 , were chosen randomly.

Considerable interference was produced by the intervening tones, even though they could be ignored (Table 1). However, the intervening spoken numbers caused only a minimum decrement in the same pitchrecognition task, both when the numbers could be

Table 1. Percent errors in the different experimental conditions. A score of 50 percent represents chance guessing in the tone-recognition task. Number recall was judged correct in any trial only if all the numbers were recalled in order.

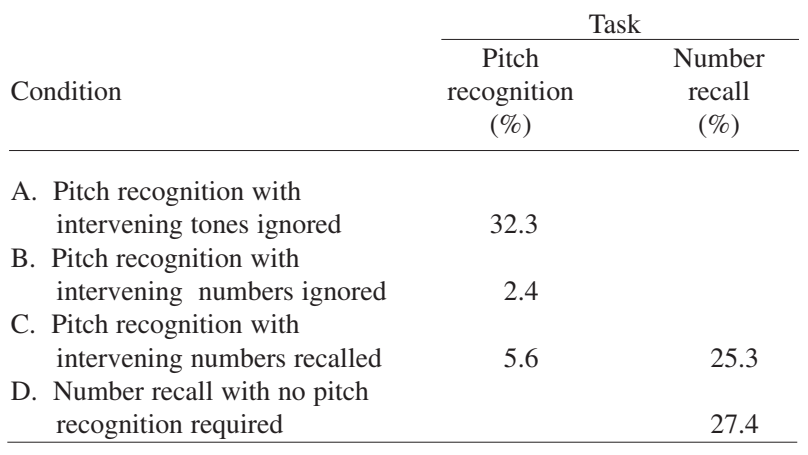


ignored and when recall of the numbers was required (Table 1). The number of errors in condition A was significantly greater than in conditions $\mathrm{B}$ or $\mathrm{C}$ on sign tests $(P<.001)$. It might be thought that such a lack of interference in condition $\mathrm{C}$ could have been obtained only at the cost of a reduced score in number recall. However, there was no significant difference in number recall between conditions $\mathrm{C}$ and D. (In fact, there were slightly fewer errors in number recall in condition $\mathrm{C}$ than in condition D.)

Thus, memory for tonal pitch is considerably disrupted by other tones. However, since the requirement to remember numbers spoken at equal loudness produces only minimum decrement in the identical pitch-recognition task, this disruption could not be due to general factors such as prevention of rehearsal, limitation in information storage capacity, or displacement in a short-term memory store in which all items or components of items are given equal weight.

Many of the experimental subjects spontaneously expressed surprise at the ease and clarity with which they could handle the verbal and the pitch information simultaneously. The distraction of attention from the pitch of the first test tone, which necessarily accompanied memorization of the numbers, caused subjectively little strain on the pitch recognition task. This was placed in sharp contrast to the subjective obliteration of memory of pitch which occurred when other tones were played.

A subsidiary conclusion concerns our mode of storage of musical information. Since memory for tonal pitch deteriorates rapidly in the presence of other tones, even in the traditional musical scale, it is most unlikely that we remember musical sequences by storing the absolute pitches of the component tones. Rather, it appears that we must rapidly discard absolute pitch information and store musical sequences in a recoded form. How this might be achieved is discussed in detail elsewhere (6).

DIANA DEUTSCH

Center for Human Information Processing, University of California, San Diego,

P. O. Box 109, La Jolla, California 92037

\section{References and Notes}

1. D. E. Broadbent, Perception and Communication (Pergamon Press, London, 1958).

2. E. Averbach and A. S. Coriell, Bell Syst. Tech. J. 40, 309 (1961).

3. R. N. Haber, Ed., Information Processing Approaches to Visual Perception. (Holt, Rinehart and Winston, New York, 1969).

4. M. I. Posner, S. J. Boies, W. H. Eichelman, and R. L. Taylor, J. Exp. Psychol. 79, Monograph No. 1 (1969).

5. International pitch $(\mathrm{A}=435)$. This proceeds in semitone steps and the frequencies employed here (in hertz) are as follows: C\#(274) D(290) D\#(308) E(326) F(345) F\#(366) G(388) G\#(411) A(435) A\#(461) B(488) C(517).

6. D. Deutsch, Psychol. Rev. 76, 300 (1969).

7. Supported in part by PHS training grant MH 10835 and PHS grant MH 15828-01. I thank G. Mandler for helpful comments. 